\title{
Effects of aging and uninephrectomy on renal changes in Tsukuba hypertensive mice
}

\author{
YOSUKE INUI $^{1,4}$, HIDEKI MOCHIDA $^{1}$, FUMIKO YAMAIRI $^{2}$, MIYOKO OKADA $^{3}$, \\ JUNJI ISHIDA ${ }^{4}$, AKIYOSHI FUKAMIZU ${ }^{4}$ and KENJI ARAKAWA ${ }^{1}$ \\ ${ }^{1}$ Pharmacology Research Laboratories II; ${ }^{2}$ Advanced Medical Research Laboratory, Mitsubishi Tanabe Pharma Corporation, \\ Toda, Saitama 335-8505; ${ }^{3}$ Safety Research Laboratory, Mitsubishi Tanabe Pharma Corporation, Kisarazu, Chiba 292-0818; \\ ${ }^{4}$ Life Science Center, Tsukuba Advanced Research Alliance, Graduate School of Life and Environmental Sciences, \\ University of Tsukuba, Tsukuba, Ibaraki 305-8577, Japan
}

Received December 28, 2012; Accepted February 26, 2013

DOI: $10.3892 /$ br.2013.74

\begin{abstract}
Renal dysfunction is accelerated by various factors such as hypertension, aging and diabetes. Glomerular hyperfiltration, considered one of the major risk factors leading to diabetic nephropathy, is often encountered in diabetic patients. However, the interrelationship of these risk factors during the course and development of renal dysfunction has not been fully elucidated. In this study, the effects of aging and uninephrectomy (UNx)-induced hyperfiltration on renal changes were investigated in Tsukuba hypertensive mice (THM) carrying both human renin and angiotensinogen genes. In THM, the urinary albumin/creatinine $(\mathrm{Alb} / \mathrm{Cr}$ ) ratio was elevated with age without a concomitant increase in the plasma $\mathrm{Cr}$ concentration. Moreover, the urinary neutrophil gelatinase-associated lipocalin/Cr (NGAL/Cr) ratio, the renal monocyte chemoattractant protein-1 (MCP-1) mRNA expression and the renal collagen type I $\alpha 2$ (COL1A2) mRNA expression were also increased with age. Age-related albuminuria in THM is likely caused by renal tubular damage, enhanced inflammatory response and tubulointerstitial fibrosis. Furthermore, following UNx, the urinary $\mathrm{Alb} / \mathrm{Cr}$ ratio and the plasma $\mathrm{Cr}$ concentration were increased in THM. The urinary NGAL/Cr ratio and the renal MCP-1 and COL1A2 mRNA expression were not affected by UNx. These results suggested that UNx-induced albuminuria in THM was caused by glomerular dysfunction, rather than renal tubular injury. In conclusion, this study demonstrated for the first time the effects of aging and UNx on renal changes in THM. These findings strongly reinforce the significance of applying a diversity of therapeutic approaches to the management of renal dysfunction.
\end{abstract}

Correspondence to: Yosuke Inui, Pharmacology Research Laboratories II, Mitsubishi Tanabe Pharma Corporation, 2-2-50 Kawagishi, Toda, Saitama 335-8505, Japan

E-mail: inui.yosuke@mn.mt-pharma.co.jp

Key words: renal disease, Tsukuba hypertensive mice, hypertension, aging, glomerular hyperfiltration, albuminuria

\section{Introduction}

Chronic kidney disease (CKD) is a major public health concern worldwide because of the increasing prevalence of end-stage renal failure requiring dialysis or kidney transplantation and the increased risk of morbidity and mortality due to cardiovascular diseases (1-6). Aging leads to renal structural changes and functional decline. Imai et al (7) and Coresh et al (8) suggested that the prevalence of CKD increases with age. Moreover, a significantly higher prevalence of CKD was reported in hypertensive patients compared to normotensive subjects $(8,9)$. High systemic blood pressure leads to pressure elevation in the glomerular capillaries, which is associated with renal vascular dysfunction. Elevated pressure in the glomerular capillaries results in glomerular sclerosis, increased albuminuria and decreased glomerular filtration rate (10). Furthermore, glomerular hyperfiltration is often observed during the early stages of diabetes and may contribute to the development of diabetic nephropathy $(11,12)$. Thus, the progression of CKD is affected by various risk factors such as aging, hypertension and hyperfiltration. However, the interrelationship of individual risk factors during the course of renal dysfunction has not been fully elucidated.

Tsukuba hypertensive mice (THM) are transgenic mice carrying both human renin and angiotensinogen genes. These animals are prone to developing hypertension (13-15). Kai et al $(16,17)$ observed albuminuria and glomerular sclerosis in 28-week-old THM. Thus, the THM is a useful model of hypertensive nephropathy induced by overproduction of angiotensin II.

In the present study, the effects of aging and uninephrectomy (UNx)-induced hyperfiltration on renal dysfunction were investigated in THM, as was the interrelationship of risk factors during the development of renal disease under hypertensive conditions.

\section{Materials and methods}

Animals. Male THM were purchased from RIKEN BioResource Center (Ibaraki, Japan) with the support of the National 
Bio-Resource Project of the Ministry of Education, Culture, Sports, Science and Technology of Japan. Age-matched male C57BL/6J mice, used as genetic background-matched wild-type mice (WTM), were purchased from Clea Japan Co., Ltd. (Tokyo, Japan). The mice were housed in a room maintained at $22 \pm 2^{\circ} \mathrm{C}$ with $55 \pm 5 \%$ relative humidity, exposed to a 12:12-h light-dark cycle and allowed free access to food and water. The animal experiments were performed in accordance with the ethical guidelines established by the Experimental Animal Care and Use Committee of Mitsubishi Tanabe Pharma Corporation.

UNx. THM and WTM, 22-26 weeks of age, were subjected to UNx or sham operation under sevoflurane (Maruishi Pharmaceutical Co. Ltd., Osaka, Japan) anesthesia. For UNx, the abdomen was opened through a left paramedian incision and the left kidney was exposed. The renal vessels and ureter were carefully isolated, ligated with a silk thread and the distal portions were cut. Control animals underwent sham operation. The mice were sacrificed at 6 weeks after surgery.

Sample collection. Prior to sacrifice, 24-, 36-, 48- and 60-week-old male THM and WTM, uninephrectomized THM (UNX-THM) and WTM (UNx-WTM) and sham-operated THM (sham-THM) and WTM (sham-WTM), were placed individually in metabolic cages (Shinano Manufacturing Co., Ltd., Tokyo, Japan) for 24-h urine collection. Urine samples were centrifuged at $1,000 \mathrm{x} \mathrm{g}$ for $10 \mathrm{~min}$ at $20^{\circ} \mathrm{C}$ and the supernatants were stored at $-20^{\circ} \mathrm{C}$ until analysis. The mice were anesthetized with sevoflurane and sacrificed by whole blood collection from the abdominal aorta. Blood samples were centrifuged at $3,000 \mathrm{x} \mathrm{g}$ for $5 \mathrm{~min}$ at $4^{\circ} \mathrm{C}$ and the plasma was separated and stored at $-80^{\circ} \mathrm{C}$ until analysis. Immediately after the animals were sacrificed, the whole kidneys of 24-, 36-, 48- and 60-week-old THM and WTM were isolated and stored in RNAlater ${ }^{\circledR}$ solution (Life Technologies, Inc., Gaithersburg, MD, USA) at $-80^{\circ} \mathrm{C}$ until RNA extraction and analysis. In the UNx experiment, approximately half of the right kidneys were isolated and stored in RNAlater solution, then stored at $-80^{\circ} \mathrm{C}$ until analysis. Part of the remaining halves of the right kidneys were fixed with $2.5 \%$ glutaraldehyde in phosphate buffer, post-fixed in $1 \%$ osmium tetroxide and embedded in epoxy resin blocks for examination under an electron microscope. The remaining parts of the right kidneys were fixed with $10 \%$ formalin and embedded in paraffin blocks for staining with periodic acid-Schiff's base.

Measurement of markers of renal function. Urinary albumin (Alb) and neutrophil gelatinase-associated lipocalin (NGAL) concentrations were determined by the Mouse Albumin ELISA kit (Shibayagi Co., Ltd., Gunma, Japan) and Mouse Lipocalin-2/NGAL Quantikine ELISA kit (R\&D Systems Inc., Minneapolis, MN, USA), respectively. Urinary and plasma creatinine (Cr) concentrations were measured by Jaffe's method using the Creatinine-Test-Wako (Wako Pure Chemical Industries, Ltd., Osaka, Japan). Alb and NGAL excretion were expressed as the $\mathrm{Alb} / \mathrm{Cr}$ and NGAL/Cr ratios, respectively.

Measurement of mRNA expression. Total RNA was isolated from the kidneys using TRIzol ${ }^{\circledR}$ reagent (Life Technologies, Inc.). Reverse transcription to cDNA of $1 \mu \mathrm{g}$ of total RNA from each sample was performed using the High Capacity RNA-to-cDNA kit (Life Technologies, Inc.). Real-time polymerase chain reaction (PCR) was performed on a 7500 Fast Real-Time PCR system (Life Technologies, Inc.), using specific TaqMan Gene Expression assays for monocyte chemoattractant protein-1 (MCP-1) (Mm00441242_m1) and renal collagen type I $\alpha 2$ (COL1A2) (Mm00483888_m1) (Life Technologies, Inc.), according to the manufacturer's instructions. Expression levels of target genes were normalized to the levels of 18S rRNA which was measured with the TaqMan ${ }^{\circledR}$ Ribosomal RNA Control Reagents kit (Life Technologies, Inc.).

Histology and electron microscopy. The glomerular sclerosis index (GSI) was calculated according to the method previously described by Simokama et al (18). Glomerular lesions were graded from 0 to $4+$ on the basis of the extent of glomerular damage and evidence of tuft involvement, as follows: 0 , no evidence of lesions; $1+, \leq 25 \%$ of tufts affected by sclerosis; $2+$, tuft involvement of 25-50\%; 3+, tuft involvement of 50-75\%; $4+$, almost complete tuft involvement. For each kidney, the sum of the results for 20 glomeruli was defined as the GSI. The structural integrity of the glomerular filtration barrier was confirmed by examination under an electron microscope. Semi-thin sections from each epoxy resin block, stained with toluidine blue, were used to select areas for electron microscopic examination. Ultra-thin sections were stained with uranyl acetate and lead citrate and examined under an H-7500 electron microscope (Hitachi, Ltd., Tokyo, Japan).

Statistical analysis. Values were presented as the mean \pm standard error of the mean (SEM), unless otherwise stated. The results were analyzed using GraphPad Prism version 5.02 (GraphPad Software, Inc., San Diego, CA, USA). Linear regression analysis was performed to calculate the slope of the age-related changes in THM and WTM. Analysis of variance was used to compare the slopes between THM and WTM. The significance of differences between UNx- and sham-THM, or UNx- and sham-WTM, was evaluated using the Student's t-test. Probabilities $<5 \%(\mathrm{P}<0.05)$ were considered to indicate a statistically significant difference.

\section{Results}

Effects of aging on glomerular and tubular injury in THM. The urinary $\mathrm{Alb} / \mathrm{Cr}$ and NGAL/Cr ratios in 60-week-old THM were higher than those in 60 -week-old WTM $(785.5 \pm 167.1$ vs. $97.5 \pm 55.5 \mu \mathrm{g} / \mathrm{mg}$ and $802.2 \pm 194.6$ vs. $65.8 \pm 9.2 \mathrm{mg} / \mathrm{mg}$, respectively). THM and WTM exhibited a linear increase in the urinary $\mathrm{Alb} / \mathrm{Cr}$ ratio with age, with a slope $[95 \%$ confidence interval $(\mathrm{CI})]$ of $17.8(10.1-25.6)\left(\mathrm{r}^{2}=0.363, \mathrm{P}<0.01\right)$ and $2.45(0.267-4.63)\left(r^{2}=0.123, P<0.05\right)$, respectively. The slope of THM was significantly steeper than that of WTM (Fig. 1A and B). In addition, a linear increase in the urinary $\mathrm{NGAL} / \mathrm{Cr}$ ratio with age was also demonstrated by a slope (95\% CI) of $18.4(10.4-26.4)\left(r^{2}=0.365, \mathrm{P}<0.01\right)$ for THM and $1.11(0.395-1.82)\left(\mathrm{r}^{2}=0.212, \mathrm{P}<0.01\right)$ for $\mathrm{WTM}$. The age-related increase in the urinary $\mathrm{NGAL} / \mathrm{Cr}$ ratio was more pronounced in THM (Fig. 1C and D). As shown in Fig. 2A and B, a more marked increase in renal MCP-1 mRNA expression with age was observed in THM, compared to that in WTM. Renal 
A

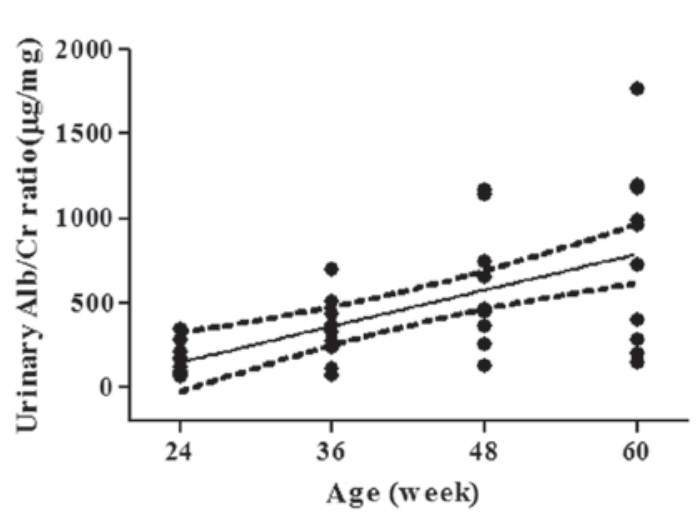

C

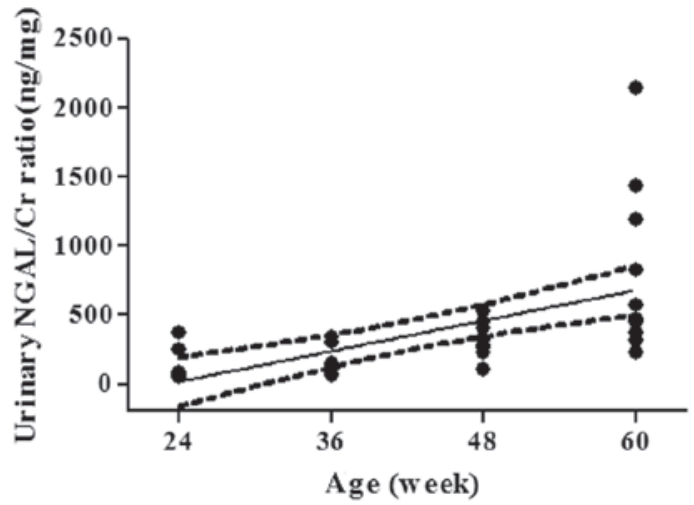

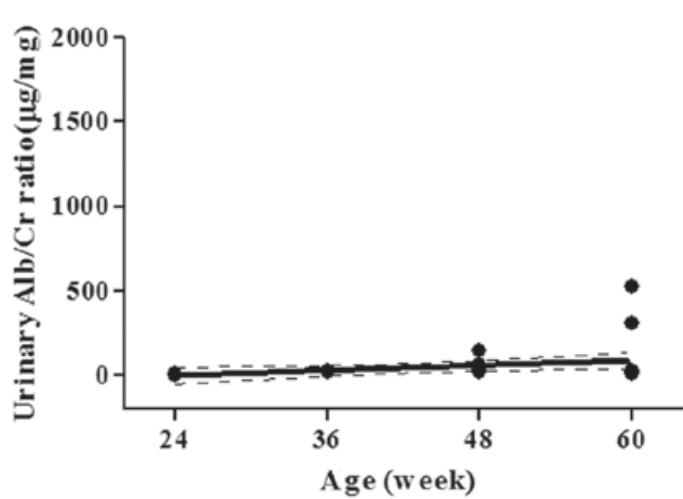

D

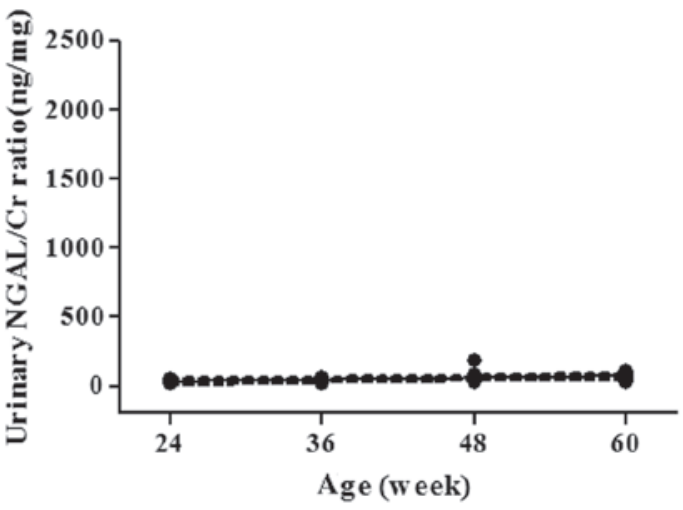

Figure 1. Effect of aging on urinary albumin/creatinine ( $\mathrm{Alb} / \mathrm{Cr}$ ) and neutrophil gelatinase-associated lipocalin (NGAL)/Cr ratios in Tsukuba hypertensive mice (THM) and wild-type mice (WTM) ( $\mathrm{n}=9-10)$. Urinary Alb/Cr ratio: (A) THM: slope (95\% CI) 17.8 (10.1-25.6), $\mathrm{r}^{2}=0.363$, P<0.01 and (B) WTM: slope (95\% CI) 2.45 (0.267-4.63), $\mathrm{r}^{2}=0.123, \mathrm{P}<0.05$. Urinary NGAL/Cr ratio: (C) THM: slope (95\% CI) 18.4 (10.4-26.4), $\mathrm{r}^{2}=0.365$, P<0.01 and (D) WTM: slope (95\% CI) 1.11 (0.395-1.82), $\mathrm{r}^{2}=0.212, \mathrm{P}<0.01$. Solid line: regression line; dotted lines: $95 \%$ CI of the slopes of the regression line. Significant differences were observed in the slopes of the urinary $\mathrm{Alb} / \mathrm{Cr}$ ratio and NGAL/Cr ratio between THM and WTM $(\mathrm{P}<0.01)$.

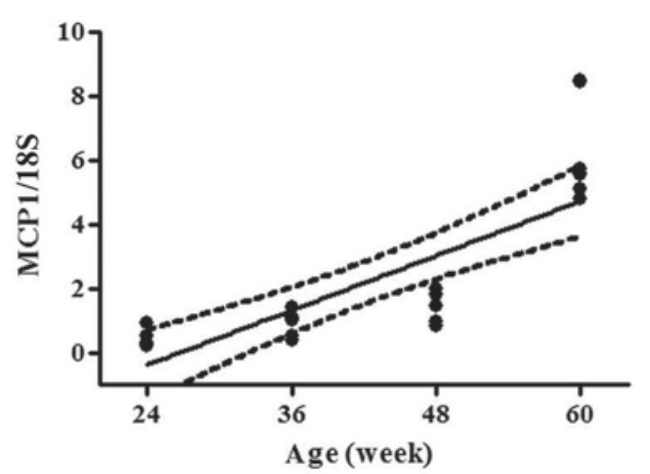

$\mathbf{C}$

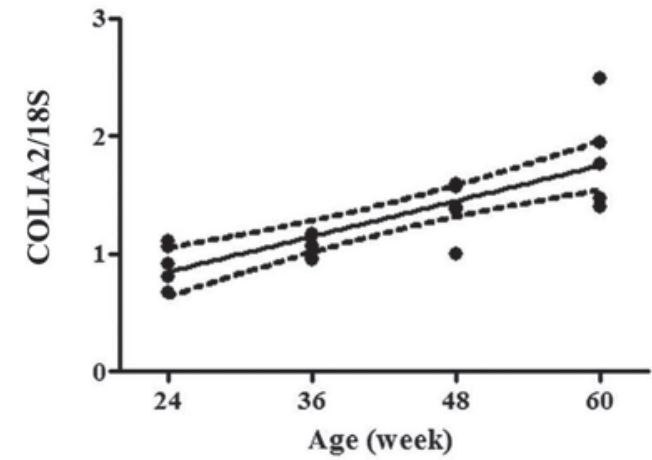

B

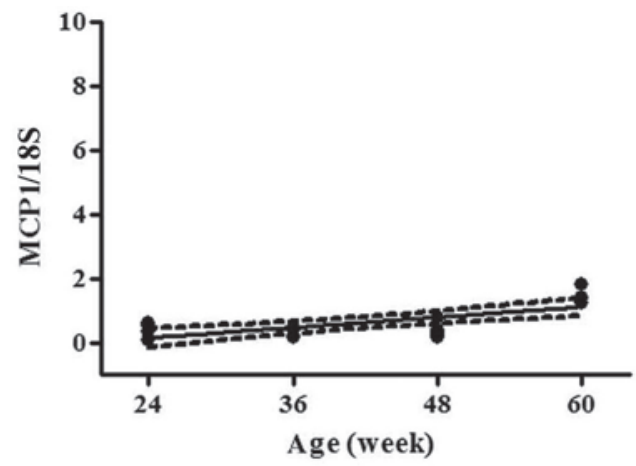

D

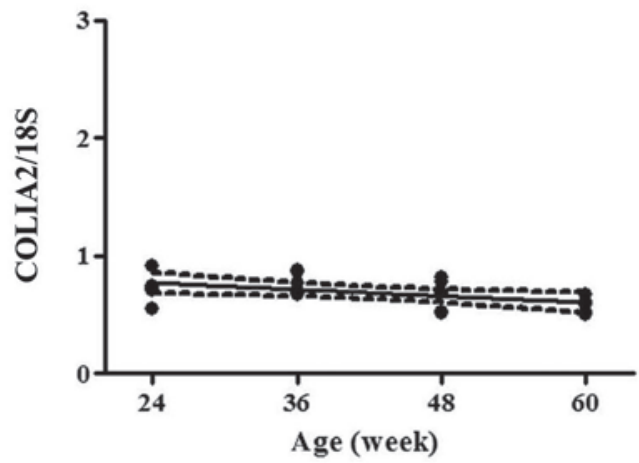

Figure 2. Effect of aging on renal monocyte chemoattractant protein-1 (MCP-1) and collagen type I $\alpha 2$ (COL1A2) expression in Tsukuba hypertensive mice (THM) and wild-type mice (WTM) (n=5). MCP-1 expression: (A) THM: slope (95\% CI) 0.142 (0.0925-0.191), $\mathrm{r}^{2}=0.671, \mathrm{P}<0.01$ and (B) WTM: slope (95\% CI) 0.0267 (0.0137-0.0397), $\mathrm{r}^{2}=0.510, \mathrm{P}<0.01$. COL1A2 expression: (C) THM: slope (95\% CI) 0.0251 (0.0159-0.0343), $\mathrm{r}^{2}=0.647$, P<0.01 and (D) WTM: slope $(95 \%$ CI $)-0.00463(-0.00857$ to -0.000695$), \mathrm{r}^{2}=0.254, \mathrm{P}<0.05$. Solid line: regression line; dotted lines: $95 \% \mathrm{CI}$ of the slopes of the regression line. Significant differences were observed in slopes of the expression of MCP-1 and COL1A2 mRNA between THM and WTM $(\mathrm{P}<0.01)$. 


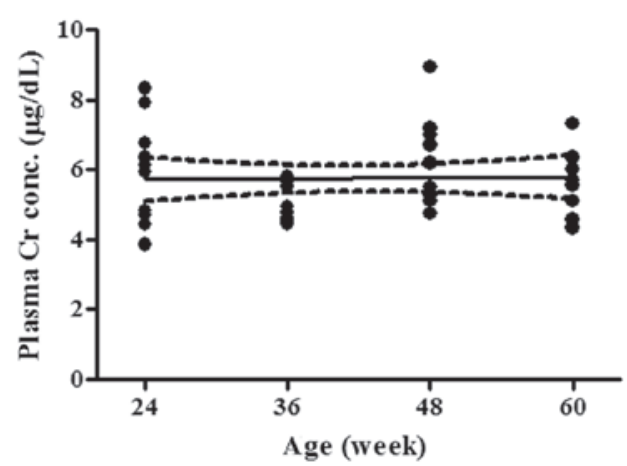

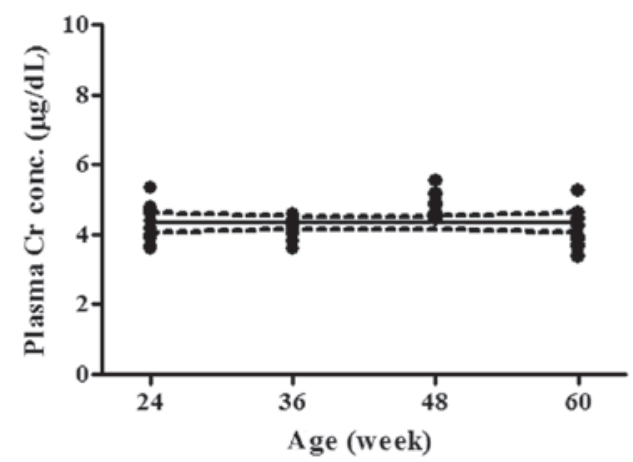

Figure 3. Effect of aging on plasma creatinine (Cr) concentration in (A) Tsukuba hypertensive mice (THM) and (B) wild-type mice (WTM) (n=10). THM: $\mathrm{P}=0.890$ and wild-type mice (WTM): $\mathrm{P}=0.975$. Solid line: regression line; dotted lines: $95 \%$ confidence interval (CI) of the slopes of the regression line. No significant difference was observed in the slopes between THM and WTM.

COL1A2 mRNA expression increased with age in THM, but not in WTM (Fig. 2C and D). Plasma Cr concentration, a marker of glomerular dysfunction, was not affected by age in THM or WTM (Fig. 3A and B).

Effects of UNx on glomerular and tubular injury in THM. The urinary $\mathrm{Alb} / \mathrm{Cr}$ ratio in UNx-THM was significantly higher than that in sham-THM (Fig. 4A). By contrast, the urinary NGAL/Cr ratio was unaffected by UNx in THM (Fig. 4B). In WTM, the urinary $\mathrm{Alb} / \mathrm{Cr}$ ratio was slightly decreased following UNx (Fig. 4A). The urinary NGAL/Cr ratio was unaffected by UNx (Fig. 4B). Expression of renal MCP-1 and COL1A2 mRNA was similar between UNx and sham animals in both THM and WTM (Fig. 5A and B).

The plasma $\mathrm{Cr}$ concentration was significantly increased in UNx-THM, but not in UNx-WTM (Fig. 6). However, GSI did not increase following UNx in THM (Fig. 7A) and there were no significant differences in the structure of the podocytes and foot processes, slit diaphragms or glomerular basement membrane (GBM) thickness between UNx-THM and sham-THM (Fig. 7B).

\section{Discussion}

As mentioned above, various risk factors, such as hypertension, aging and hyperfiltration, affect the progression of CKD $(1,11,19,20)$. In the present study, the interrelationship of these risk factors was investigated. The results indicated that aging and UNx exerted a similar effect on the development of albuminuria in THM. Of note, although the urinary NGAL/Cr ratio and renal MCP-1 and COL1A2 mRNA expression were increased with age in THM, these alterations were not observed in UNx-THM. Plasma Cr concentration was elevated by UNx only in THM. These results demonstrated that age- and UNx-related renal changes differed on a qualitative level.

Albuminuria may result from changes in glomerular filtration, as well as from reduced reabsorption of filtered albumin by the proximal tubule $(21,22)$. Therefore, the effect of aging on the urinary NGAL/Cr ratio was investigated. In THM, the urinary NGAL/Cr ratio increased with age. There was no effect of aging on plasma NGAL levels in THM (data not shown) and the reabsorption of NGAL by the tubular epithelial cells after
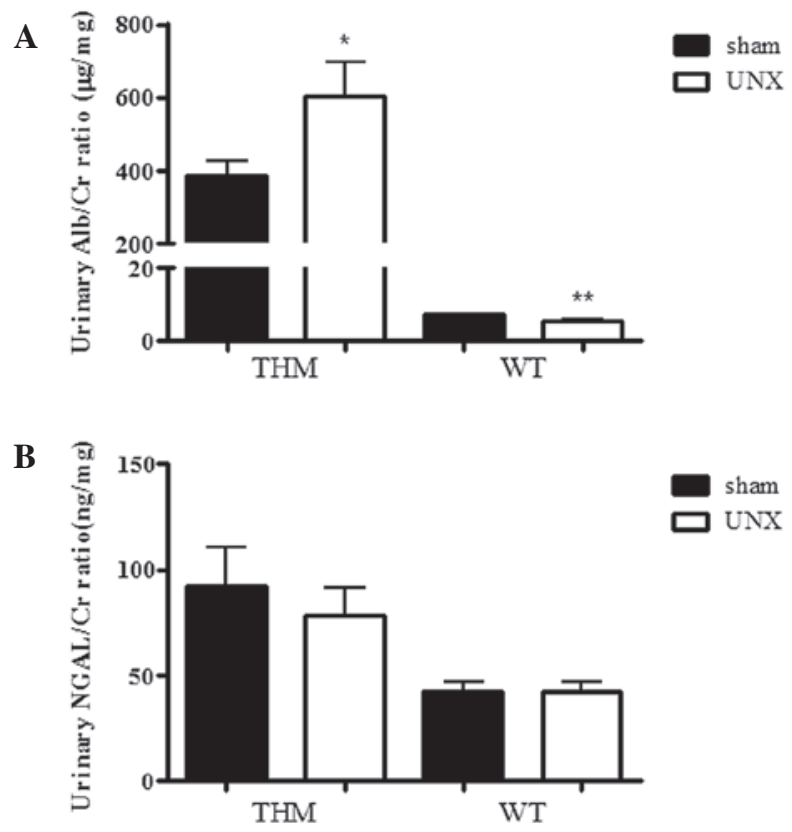

Figure 4. Effect of uninephrectomy (UNx) on (A) urinary albumin/creatinine $(\mathrm{Alb} / \mathrm{Cr})$ ratio and $(\mathrm{B})$ urinary neutrophil gelatinase-associated lipocalin (NGAL)/Cr ratio in Tsukuba hypertensive mice (THM) and wild-type mice (WTM) $(n=9-10)$. Data are expressed as the means \pm standard error of the mean (SEM). ${ }^{*} \mathrm{P}<0.05,{ }^{* *} \mathrm{P}<0.01$ vs. sham-operated mice.
$\mathbf{A}$

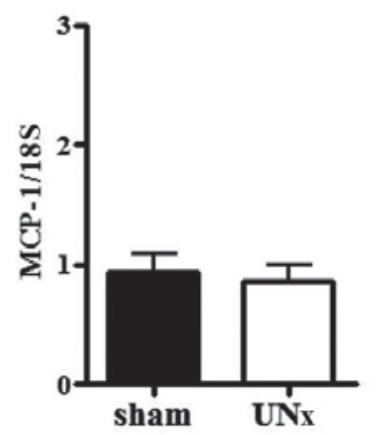

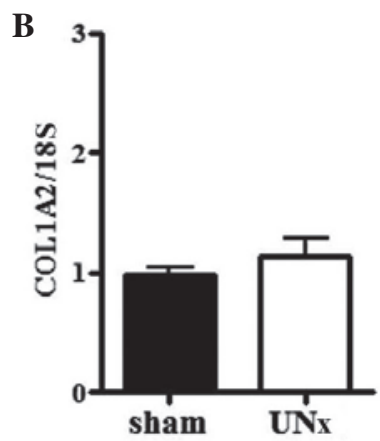

Figure 5. Effect of uninephrectomy (UNx) on (A) monocyte chemoattractant protein-1 (MCP-1) and (B) collagen type I $\alpha 2$ (COL1A2) expression in the kidneys of Tsukuba hypertensive mice (THM) ( $\mathrm{n}=4$ and 5 , respectively). Expression of both was normalized with $18 \mathrm{~S}$ ribosomal RNA. Data are expressed as the means \pm standard error of the mean (SEM). 


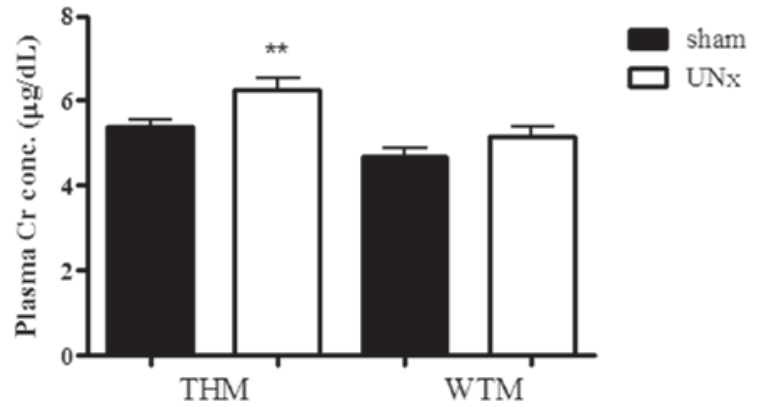

Figure 6. Effect of uninephrectomy (UNx) on plasma creatinine (Cr) concentration in Tsukuba hypertensive mice (THM) and wild-type mice (WTM) $(n=9-10)$. Data are expressed as the means \pm standard error of the mean (SEM). ${ }^{* *} \mathrm{P}<0.01$ vs. sham-THM.

A

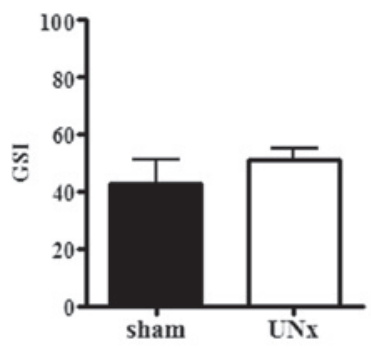

B

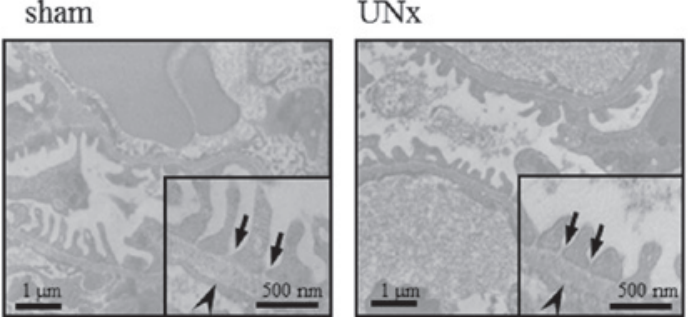

Figure 7. Effect of uninephrectomy (UNx) on glomerular sclerosis and filtration barrier in Tsukuba hypertensive mice (THM). (A) Effect of UNx on glomerular sclerosis index (GSI) score in THM $(n=10)$. Data are expressed as the means \pm standard error of the mean (SEM). (B) Electron micrographs of typical glomerular filtration barrier in sham- and UNx-THM. Arrows: slit diaphragms; arrowheads: glomerular basement membrane (GBM).

glomerular filtration has been well-characterized (23). Thus, the present study has demonstrated that urinary NGAL may be considered as a biomarker of tubular damage. These observations suggest that the development of albuminuria in THM was accelerated by aging and that age-related renal changes were induced by renal tubular damage.

In the kidney, MCP-1 plays an important role in recruiting monocytes/macrophages to the injured tubulointerstitial tissue (24). Furthermore, COL1A2 is associated with tubulointerstitial fibrosis (25). In this study, the levels of renal MCP-1 and COL1A2 mRNA expression increased with age in THM. Renal tubular inflammation and tubulointerstitial fibrosis contributed to the progression of renal tubular damage and renal dysfunction $(26,27)$. Thus, the results of this study have demonstrated that the age-related albuminuria observed in THM was caused by renal tubular damage.

Shimokama et al (18) reported moderate focal segmental glomerular sclerosis in THM. However, this study did not demonstrate an increase in plasma $\mathrm{Cr}$ concentration with age in THM. In addition, aging exerted no effects on the glomerular filtration barrier (data not shown). Taken together, these observations strongly suggest that age-related albuminuria in THM was mainly caused by renal tubular damage rather than glomerular dysfunction.

UNx induces glomerular hyperfiltration and is associated with albuminuria in experimental animal models (28). An increased urinary $\mathrm{Alb} / \mathrm{Cr}$ ratio was observed following UNx in THM in the present study. However, the urinary NGAL/Cr ratio and renal MCP-1 and COL1A2 mRNA levels were unaffected by UNx, indicating that UNx-related renal changes differed from those induced by aging. Moreover, these results demonstrated that UNx-induced albuminuria was not caused by renal tubular injury.

In the present study, the plasma $\mathrm{Cr}$ concentration was increased following UNx in THM, suggesting that glomerular dysfunction developed in UNx-THM. The glomerular filtration barrier is formed by three layers: the innermost fenestrated vascular endothelium, the GBM and the podocyte layer (29). Glomerular size selectivity is maintained by the GBM and the slit pore diaphragm between the foot processes of podocytes (29). However, no progression of glomerular sclerosis, loss of slit diaphragms or increased GBM thickness was observed when comparing UNx-THM to sham-THM. Therefore, UNx exerted no effect on the integrity of the glomerular filtration barrier and the UNx-induced albuminuria in THM may not be attributed to changes in glomerular size selectivity.

Charge selectivity is attributed to the anionic sites of the glomerular endothelial surface layer (ESL) (30). Loss of the glomerular ESL has been associated with albuminuria in patients with diabetic nephropathy and in an experimental animal model $(31,32)$. In the present study, the loss of anionic charges in the GBM and ESL was not assessed in UNx-THM. Therefore, further investigations are required to elucidate the involvement of a charge-selective glomerular filtration barrier in renal dysfunction of UNX-THM.

In conclusion, this study has demonstrated that aging and hyperfiltration accelerated the development of albuminuria in hypertensive mice and that these renal changes differed in origin. Age-related albuminuria was mainly caused by renal tubular damage and UNx-induced albuminuria was due to glomerular dysfunction. These findings indicate that the pathophysiology of CKD is complicated. A diversity of therapeutic approaches must be applied to the treatment of renal dysfunction to address the possible implicated factors.

\section{Acknowledgements}

The authors would like to thank Dr M. Nagasaki, Dr A. Umeda, Dr R. Yamauchi, Dr H. Doi, Dr K. Kikkawa, Dr M. Nishio, Mr. Y. Egi, Mr. K. Fujitaka, Ms. Y. Ogawa, Mr. K. Fujiki and Ms. A. Yamazaki of Mitsubishi Tanabe Pharma Corporation for their support and for their help with the statistical analysis.

\section{References}

1. Levey AS, Eckardt KU, Tsukamoto Y, et al: Definition and classification of chronic kidney disease: a position statement from Kidney Disease: Improving Global Outcomes (KDIGO). Kidney Int 67: 2089-2100, 2005. 
2. Jungers P: Screening for renal insufficiency: is it worth while? is it feasible? Nephrol Dial Transplant 14: 2082-2084, 1999.

3. Akiba T, Nakai S, Shinzato T, et al: Why has the gross mortality of dialysis patients increased in Japan? Kidney Int 57: S60-S65, 2000.

4. Weiner DE, Tighiouart H, Amin MG, et al: Chronic kidney disease as a risk factor for cardiovascular disease and all-cause mortality: a pooled analysis of community-based studies. J Am Soc Nephrol 15: 1307-1315, 2004

5. Jafar TH, Stark PC, Schmid CH, et al: Progression of chronic kidney disease: the role of blood pressure control, proteinuria and angiotensin-converting enzyme inhibition: a patient-leve meta-analysis. Ann Intern Med 139: 244-252, 2003.

6. Iseki K, Ikemiya Y, Iseki C and Takishita S: Proteinuria and the risk of developing end-stage renal disease. Kidney Int 63 : $1468-1474,2003$.

7. Imai E, Horio M, Iseki K, et al: Prevalence of chronic kidney disease (CKD) in the Japanese general population predicted by the MDRD equation modified by a Japanese coefficient. Clin Exp Nephrol 11: 156-163, 2007.

8. Coresh J, Astor BC, Greene T, Eknoyan G and Levey AS Prevalence of chronic kidney disease and decreased kidney function in the adult US population: Third National Health and Nutrition Examination Survey. Am J Kidn Dis 41: 1-12, 2003.

9. Tozawa M, Iseki K, Iseki C, Kinjo K, Ikemiya $Y$ and Takishita S Blood pressure predicts risk of developing end-stage renal disease in men and women. Hypertension 41: 1341-1345, 2003.

10. Feld LG, Van Liew JB, Galaske RG and Boylan JW: Selectivity of renal injury and proteinuria in the spontaneously hypertensive rat. Kidney Int 12: 332-343, 1977.

11. Levine DZ: Can rodent models of diabetic kidney disease clarify the significance of early hyperfiltration?: recognizing clinical and experimental uncertainties. Clin Sci (Lond) 114: 109-118, 2008.

12. Yamagata K, Ishida K, Sairenchi T, et al: Risk factors for chronic kidney disease in a community-based population: a 10-year follow-up study. Kidney Int 71: 159-166, 2007.

13. Fukamizu A, Sugimura K, Takimoto E, et al: Chimeric renin-angiotensin system demonstrates sustained increase in blood pressure of transgenic mice carrying both human renin and human angiotensinogen genes. J Biol Chem 268: 11617-11621, 1993.

14. Fukamizu A, Seo MS, Hatae T, et al: Tissue-specific expression of the human renin gene in transgenic mice. Biochem Biophys Res Commun 165: 826-832, 1989.

15. Takahashi S, Fukamizu A, Hasegawa T, et al: Expression of the human angiotensinogen gene in transgenic mice and transfected cells. Biochem Biophys Res Commun 180: 1103-1109, 1991.

16. Kai T, Sugimura K, Shimada S, Kurooka A, Takenaka T and Ishikawa K: Inhibitory effects of a subdepressor dose of L-158,809, an angiotensin II type 1 receptor antagonist, on cardiac hypertrophy and nephropathy via the activated human renin-angiotensin system in double transgenic mice with hypertension. Jpn Circ J 62: 599-603, 1998.

17. Kai T, Kino H and Ishikawa K: Role of the renin-angiotensin system in cardiac hypertrophy and renal glomerular sclerosis in transgenic hypertensive mice carrying both human renin and angiotensinogen genes. Hypertens Res 21: 39-46, 1998.
18. Shimokama T, Haraoka S, Horiguchi H, Sugiyama F, Murakami K and Watanabe T: The Tsukuba hypertensive mouse (transgenic mouse carrying human genes for both renin and angiotensinogen) as a model of human malignant hypertension: development of lesions and morphometric analysis. Virchows Archiv 432: 169-175, 1998.

19. Hsu CY, Iribarren C, McCulloch CE, Darbinian J and Go AS: Risk factors for end-stage renal disease: 25 -year follow-up. Arch Inter Med 169: 342-350, 2009.

20. Helal I, Fick-Brosnahan GM, Reed-Gitomer B and Schrier RW: Glomerular hyperfiltration: definitions, mechanisms and clinical implications. Nat Rev Nephrol 8: 293-300, 2012.

21. Russo LM, Sandoval RM, Campos SB, Molitoris BA, Comper WD and Brown D: Impaired tubular uptake explains albuminuria in early diabetic nephropathy. J Am Soc Nephrol 20: 489-494, 2009.

22. Comper WD, Haraldsson B and Deen WM: Resolved: normal glomeruli filter nephrotic levels of albumin. J Am Soc Nephrol 19: 427-432, 2008

23. Birn H and Christensen EI: Renal albumin absorption in physiology and pathology. Kidney Int 69: 440-449, 2006.

24. Wada T, Furuichi K, Sakai N, et al: Up-regulation of monocyte chemoattractant protein-1 in tubulointerstitial lesions of human diabetic nephropathy. Kidney Int 58: 1492-1499, 2000.

25. Korrapati MC, Shaner BE, Neely BA, Alge JL, Arthur JM and Schnellmann RG: Diabetes-induced renal injury in rats is attenuated by suramin. J Pharmacol Exp Ther 343: 34-43, 2012.

26. Eardley KS, Zehnder D, Quinkler M, et al: The relationship between albuminuria, MCP-1//CCL2, and interstitial macrophages in chronic kidney disease. Kidney Int 69: 1189-1197, 2006.

27. Taniguchi H, Kojima R, Sade H, Furuya M, Inomata $\mathrm{N}$ and Ito $\mathrm{M}$ : Involvement of MCP-1 in tubulointerstitial fibrosis through massive proteinuria in anti-GBM nephritis induced in WKY rats. J Clin Immunol 27: 409-429, 2007.

28. Hostetter TH, Olson JL, Rennke HG, Venkatachalam MA and Brenner BM: Hyperfiltration in remnant nephrons: a potentially adverse response to renal ablation. Am J Physiol 241: F85-F93, 1981.

29. Kanwar YS, Danesh FR and Chugh SS: Contribution of proteoglycans towards the integrated functions of renal glomerular capillaries: a historical perspective. Am J Pathol 171: 9-13, 2007.

30. Haraldsson B, Nyström J and Deen WM: Properties of the glomerular barrier and mechanisms of proteinuria. Physiol Rev 88: 451-487, 2008.

31. Broekhuizen LN, Lemkes BA, Mooij HL, et al: Effect of sulodexide on endothelial glycocalyx and vascular permeability in patients with type 2 diabetes mellitus. Diabetologia 53: 2646-2655, 2010

32. Salmon AHJ, Ferguson JK, Burford JL, et al: Loss of the endothelial glycocalyx links albuminuria and vascular dysfunction. J Am Soc Nephrol 23: 1339-1350, 2012. 